\title{
Transparent polymer solar cells employing a layered light-trapping architecture
}

\author{
Rafael Betancur ${ }^{1 \dagger}$, Pablo Romero-Gomez ${ }^{1 \dagger}$, Alberto Martinez-Otero ${ }^{1}$, Xavier Elias', Marc Maymó1 \\ and Jordi Martorell1,2ᄎ
}

\begin{abstract}
Organic solar cells have unique properties that make them very attractive as a renewable energy source. Of particular interest are semi-transparent cells, which have the potential to be integrated into building façades yet not completely block light. However, making organic cells transparent limits the metal electrode thickness to a few nanometres, drastically reducing its reflectivity and the device photon-harvesting capacity. Here, we propose and implement an ad hoc path for light-harvesting recovery to bring the photon-to-charge conversion up to almost $80 \%$ that of its opaque counterpart. We report semi-transparent $\mathrm{PTB7:PC}{ }_{71} \mathrm{BM}$ cells that exhibit $30 \%$ visible light transmission and $5.6 \%$ power conversion efficiency. Non-periodic photonic crystals are used to trap near-infrared and near-ultraviolet photons. By modifying the layer structure it is possible to tune the device colour without significantly altering cell performance.
\end{abstract}

T he unique properties of the active material used to capture solar photons in organic photovoltaics (OPVs)-light weight, flexibility, semi-transparency, sensitivity to low light levels or non-direct sunlight, and solution-processing-make OPVs one of the most attractive photovoltaic technologies for the development of electricity production units to be integrated into everyday life. In recent years, it has been recognized by many authors that the intrinsic semi-transparency of the active material found in many OPV devices ${ }^{1-27}$ opens a route to their integration into transparent elements such as windows in buildings and automobiles or screens in electronic equipment. In most polymer cells, the low charge carrier mobility in donor or acceptor organic materials prevents the use of the thick active layers that would be needed for very efficient photon harvesting. However, such low charge mobility and the resulting associated semi-transparency of the active layer may turn out to be the strongest asset for OPVs to compete in the photovoltaic production of electrical energy. In a conventional OPV device it is not possible to make use of the active-layer semi-transparency, because the device is capped with a non-transparent metal layer that serves a dual purpose, as one of the electrodes of the cell and as a mirror to reflect non-absorbed photons in the first pass back into the device. It has not been until recently that semi-transparent top electrodes have been fabricated with electrical properties similar to those where electrodes are deposited directly on the substrate ${ }^{28-36}$. Reharvesting infrared or ultraviolet photons that are lost in semi-transparent devices requires either additional changes in the cell architecture or the use of materials with an enhanced absorption in the near-ultraviolet and especially in the near-infrared. A Bragg reflector has been used to increase near-infrared photon harvesting, demonstrating that the power conversion efficiency (PCE) of OPV cells could be increased up to $1.7 \%$ for small-molecule cells ${ }^{37}$ and up to $2.5 \%$ for polymer cells $^{38}$, and could improve transparency in thin-film a-Si:H cells ${ }^{39}$. An alternative approach is to reharvest red light using cholesteric liquid crystals as wavelength-dependent reflectors ${ }^{40}$. Enhancing near-infrared photon harvesting is also achievable by considering donor polymers with an absorption band infrared-shifted close to $800 \mathrm{~nm}$. With such a strategy, a rather significant breakthrough in semi-transparent OPVs was achieved recently, with PCE $>4 \%$ reported $^{41,42}$. The use of high-performance polymers or tandem cells has also been considered in semi-transparent cells $s^{43-45}$. In this case, either efficiencies above $5 \%$ can be achieved at the expense of a rather limited luminosity, or high transmission in the visible is obtained at the expense of reduced near-infrared photon harvesting.

An approach is needed that combines high-performance polymer photovoltaic materials with a photonic configuration to reharvest near-ultraviolet and near-infrared photons when the top electrode is thinned down, to take the PCE of visible transparent cells to the upper-limit efficiencies recently established based on Schockley-Queisser theory ${ }^{46}$. In the scientific literature, the single-junction opaque OPV cells that currently exhibit the highest efficiency are cells made with bulk heterojunctions of PTB7:PC ${ }_{71} \mathrm{BM}^{47}$. Although lowering the bandgap of such types of material is a desirable objective to increase the efficiency for transparent as well as non-transparent cells, attempts to do so have not yet yielded efficiencies higher than those measured with PTB7:PC ${ }_{71}$ BM for direct ${ }^{47,48}$ or inverted cells ${ }^{47}$. The extinction coefficient of this blend (Fig. 1a) for $300-800 \mathrm{~nm}$ averages 0.2 . With such an extinction coefficient and a 100 -nm-thick layer, approximately half the photons at any given wavelength are not absorbed during the first pass. As indicated, when the mirror effect of the back electrode is removed, photonic management becomes essential. The use of only a Bragg reflector to reflect the infrared and transmit the visible would not produce an optimal result. A periodic multilayer optimizes the interference to obtain maximum reflectivity at the wavelength satisfying the Bragg condition. In a photovoltaic device, interference must be optimal at all wavelengths for the part of the solar spectrum being absorbed by the active material. In principle, broader light management could be achieved by implementing a two- or three-dimensional nanostructuring of the refractive index. However, such a high level of nanostructuring may not be desirable in solar cells, for obvious reasons.

An alternative route to the goal of broadband photonic control using simple one-dimensional structures is to increase the degrees of freedom relative to the periodic one-dimensional structure. In 

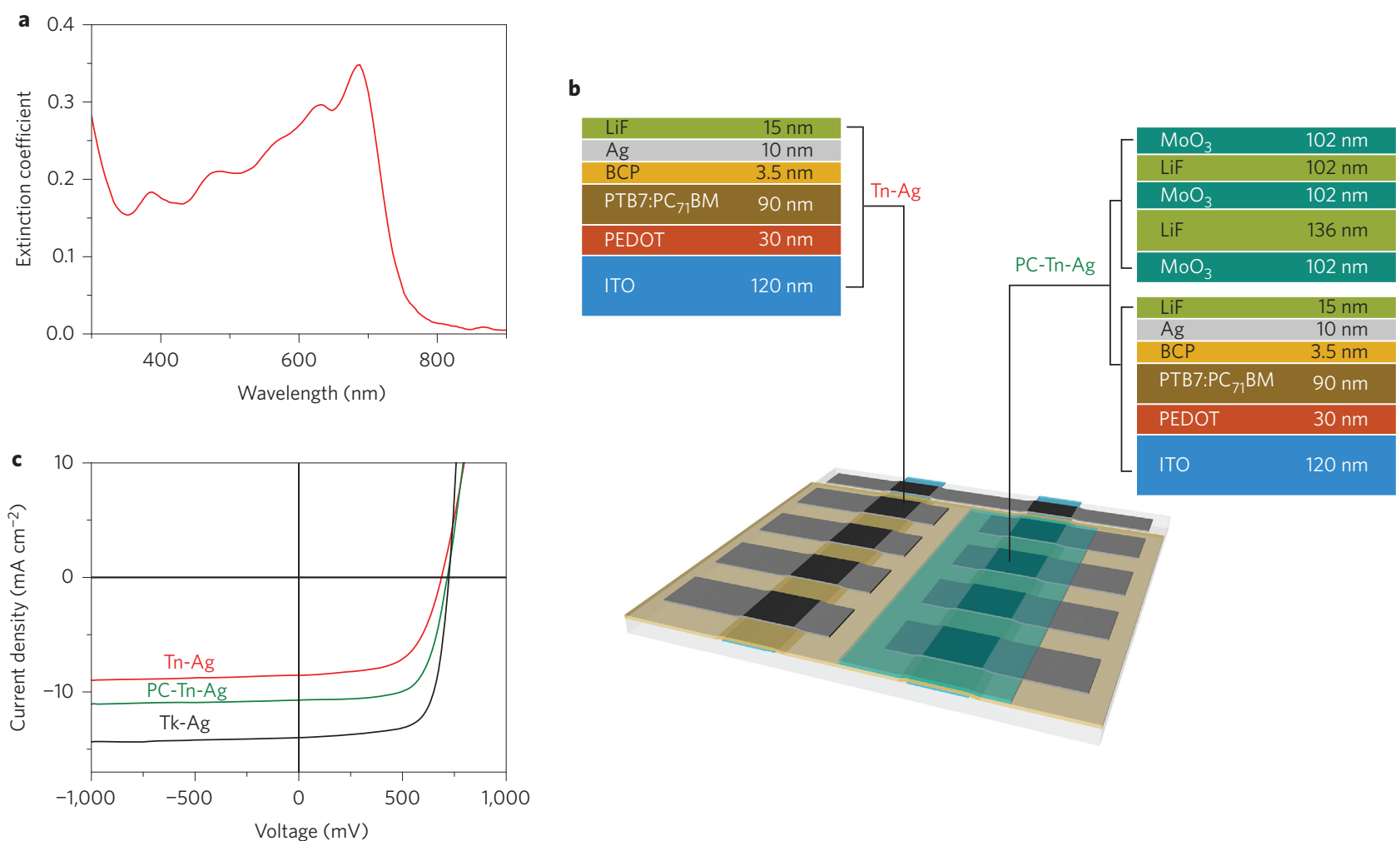

Figure 1 | Polymer blend and device. a, PTB7:PC ${ }_{71} \mathrm{BM}$ extinction coefficient. $\mathbf{b}$, Schematic view of the fabricated photovoltaic cells. Two stripes of ITO were crossed by eight stripes of silver layers defining $9 \mathrm{~mm}^{2}$ cells. The other layers between the electrodes were deposited to cover the entire substrate. A long metal stripe at the back was deposited to contact the ITO. Using a mask, the photonic crystal was grown to cover, as in the schematic, half of the devices. The layer thicknesses given for the photovoltaic part of the structure are the ones used for all semi-transparent devices in the current work. The thicknesses for the photonic-crystal layers correspond to a device for which the current density-voltage curve would be similar to the one shown in c. Layer thicknesses are not fully to scale. c, Current density-voltage curves for the Tk-Ag (black), Tn-Ag (red) and PC-Tn-Ag (green) cells fabricated with 330 nm ITO.

Table 1 | Photovoltaic parameters for the fabricated devices.

\begin{tabular}{|c|c|c|c|c|c|c|c|}
\hline & ITO & $J_{\mathrm{sc}}\left(\mathrm{mA} \mathrm{cm} \mathrm{cm}^{-2}\right)$ & Relative $J_{\mathrm{sc}}(\%)$ & $V_{\text {oc }}(\mathrm{mV})$ & FF (\%) & PCE (\%) & Luminosity (\%) \\
\hline Tk-Ag & & 14.0 & 100 & 724 & 71 & 7.3 & 0 \\
\hline $\mathrm{Tn}-\mathrm{Ag}$ & 330 nm & 8.5 & 61 & 688 & 61 & 3.6 & 32 \\
\hline PC-Tn-Ag & & 10.7 & 77 & 716 & 68 & 5.2 & 29 \\
\hline $\mathrm{Tk}-\mathrm{Ag}$ & 170 מ & 14.1 & 100 & 744 & 73 & 7.7 & 0 \\
\hline PC-Tn-Ag & $120 \mathrm{~nm}$ & 10.9 & 77 & 733 & 70 & 5.6 & 28 \\
\hline
\end{tabular}

the current work we report on the design and fabrication of a fivelayer photonic structure that provides optimal interference at each wavelength to obtain the largest efficiency while at the same time maintaining a very good transparency in most the visible wavelengths. Although the resulting multilayer structure lacks periodicity, the optical control it provides is, in many ways, similar to that provided by photonic crystals, and we will use this term to describe it throughout. Such photonic crystals must be numerically designed while noting that the photonic crystal and all other layers in the device form an integral cell architecture.

We consider two types of reference device, which we term Tk-Ag and $\mathrm{Tn}-\mathrm{Ag}$, and compare these with the device that includes the photonic crystal. The architecture of the two reference cells is the same except for the thickness of the top silver electrode and a protective $15-\mathrm{nm}$-thick LiF layer included in the Tn-Ag cell. The use of such a protective layer is necessary to extend the lifetime for such devices. Note that none of the devices used throughout the work were encapsulated. For the Tk-Ag device the thickness of the evaporated top silver electrode is $100 \mathrm{~nm}$, whereas for the Tn-Ag cell, shown schematically in Fig. 1b, the thickness of the top electrode is only $10 \mathrm{~nm}$. The active material is in all cases a 90 -nm-thick bulk heterojunction of PTB7:PC $\mathrm{P}_{71} \mathrm{BM}$ blend. As has been shown recently, a $9.5 \%$ increase in PCE can be achieved if the calcium layer, which is most commonly used as the hole-blocking layer for such cells, is replaced by a highly transparent $3.5 \mathrm{~nm}$ layer of bathocuproine $(\mathrm{BCP})^{48}$. As seen in Table 1 and Fig. 1c, the fabricated Tk$\mathrm{Ag}$ cell with $330 \mathrm{~nm}$ indium tin oxide (ITO) yielded a short-circuit current of $14 \mathrm{~mA} \mathrm{~cm}^{-2}$ and a PCE of $7.3 \%$, which are taken as the reference cell parameters for comparison with the semi-transparent cells. The experimentally measured external quantum efficiency (EQE) for the Tk-Ag cell is shown in Fig. 2a. When the thickness of the top electrode is reduced to $10 \mathrm{~nm}$, the collection of photons becomes less effective and the EQE is reduced at all wavelengths (Fig. 2a). In Fig. 3a, the cell is shown to become semi-transparent with a homogeneous transmission close to $30 \%$ in the nearultraviolet, visible and near-infrared ranges. Such a gain in transparency is quite detrimental to cell performance, particularly in the near-infrared, because this is precisely the spectral region where the number of solar photons is largest. It is worth noting that the wavelength dependence seen for the EQE of the Tn-Ag cell does not exhibit any pronounced oscillation, as the reflectivity reduction for the last electrode prevents the formation of standing 

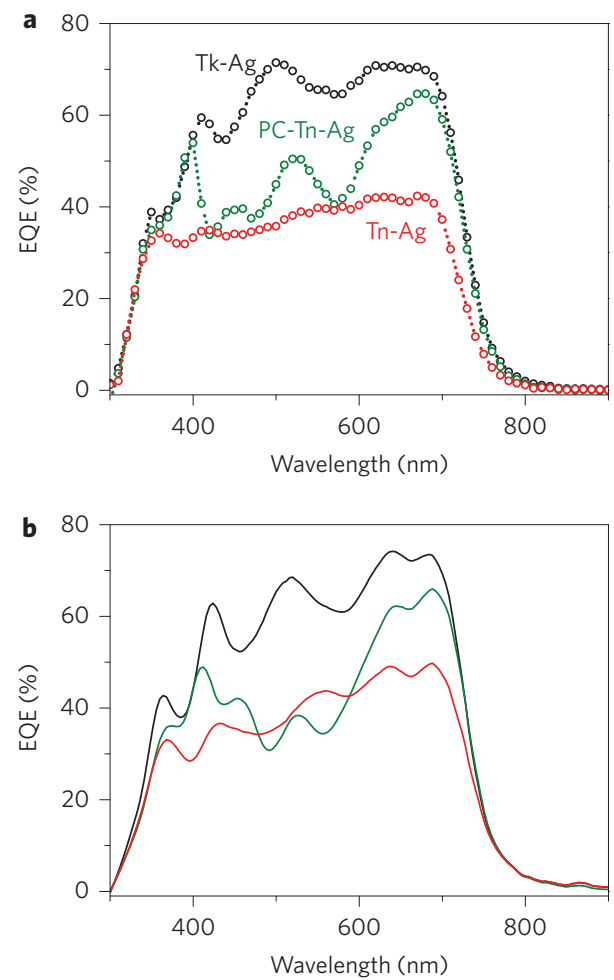

Figure 2 | External quantum efficiency. a, Experimentally measured EQEs for the opaque cell ( $\mathrm{Tk}-\mathrm{Ag}$, black circles), the semi-transparent cell where the top electrode is a 10-nm-thick silver layer ( $\mathrm{Tn}-\mathrm{Ag}$, red circles) and the cell incorporating the photonic crystal (PC-Tn-Ag, green circles). The trapping for the near-ultraviolet is very effective and the $J_{\mathrm{sc}}$ resulting from photons at wavelengths in the $300-400 \mathrm{~nm}$ range corresponds to $98 \%$ of the opaque cells. For near-infrared photons in the $650-900 \mathrm{~nm}$ range, the $J_{\text {sc }}$ corresponds to $91 \%$ that of opaque cells. b, Numerically calculated EQEs for the opaque cell (black line), the semi-transparent cell where the top electrode is a 10-nm-thick silver layer (red line) and the cell that incorporates the photonic crystal (green line).

waves. Proper light management becomes an essential issue if it is desirable to extract the largest current from such thin-film semitransparent devices.

To recover the efficiency in light harvesting but not lose transparency in the visible, ideally one would like to have a perfect mirror for the near-infrared and near-ultraviolet, and zero reflectivity in the visible. As indicated above, to achieve such a goal we consider one-dimensional photonic crystals, incorporating an additional degree of freedom by removing their inherent periodicity. The practical implementation of this typically requires the application of numerical inverse problem-solving methods to find the optimal design for the nanolayer structure, knowing the target solution. For a transparent photovoltaic window there are essentially two parameters that determine its performance: the efficiency of converting light to electricity and the device visible transmission or luminosity. The luminosity of a semi-transparent device corresponds to the integral of the transmission weighted by the product of the human eye photopic spectral response with illumination from the white standard illuminant CIE-D65 (Supplementary Section S1), normalized by the integral of the product of the photopic curve and illuminant shown in Fig. 3a.

In our numerical calculation we sought to design a photonic multilayer to maximize the contribution to $J_{\text {sc }}$ for wavelengths below $400 \mathrm{~nm}$ and above $650 \mathrm{~nm}$ and where luminosity is at least $90 \%$ of that of the Tn-Ag cell. Figure 4a shows that $J_{\text {sc }}$ increases rapidly when we increase the number of layers in the photonic
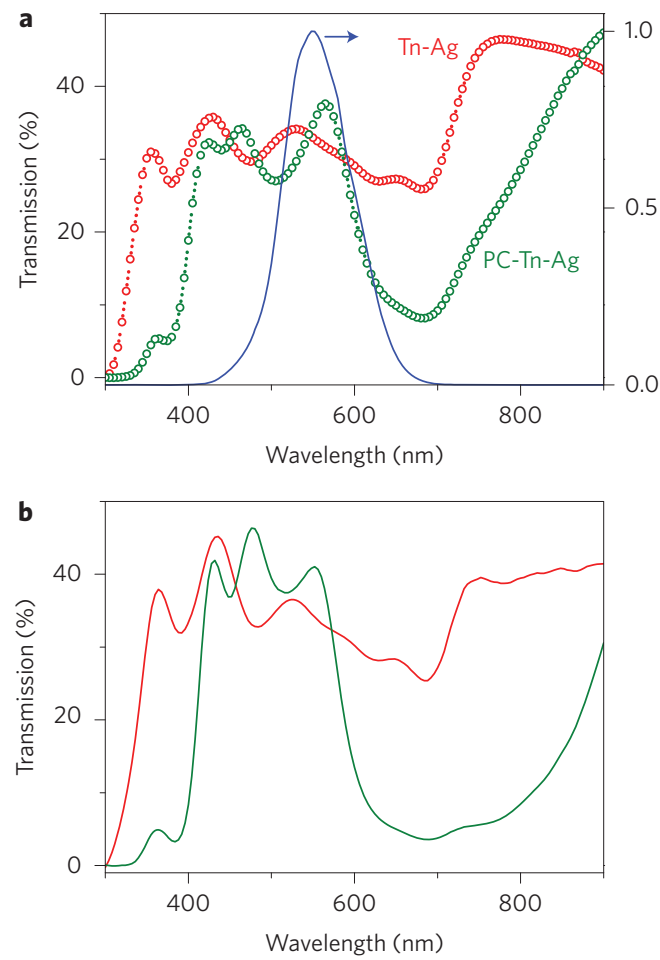

Figure 3 | Device transmission. a, Experimentally measured transmission for the semi-transparent cell where the top electrode is a 10-nm-thick silver layer ( $\mathrm{Tn}-\mathrm{Ag}$, red circles) and for the cell that incorporates the photonic crystal (PC-Tn-Ag, green circles). The product of the photopic curve and CIE-65D illuminant is normalized to one (blue line). Note that this product is relatively small in the $400-500 \mathrm{~nm}$ range. This is because the sensitivity of the human eye to blue colours is rather low. However, to cause minimal alteration to the colours of the image being observed through the PC-Tn-Ag devices, it is important to keep the same level of transparency in that wavelength range as in the 500-650 nm range. b, Numerically calculated transmission for the semi-transparent cell where the top electrode is a 10-nm-thick silver layer (red line) and for the cell that incorporates the photonic crystal (green line).

crystal, and saturates beyond five layers. Although $J_{\mathrm{sc}}$ for the Tn-Ag cell is $63.8 \%$ that of the opaque cell, the $J_{\text {sc }}$ calculated for the five-layer photonic crystal (PC)-Tn-Ag cell is $76.3 \%$ that of the opaque cell. The photonic-crystal structure we designed combines layers of a low-refractive-index material ( $\mathrm{LiF})$ with layers of highrefractive-index material $\left(\mathrm{MoO}_{3}\right)$. The increase in $J_{\mathrm{sc}}$ from a periodic or Bragg structure was also calculated (Fig. 4a). For a Bragg reflector of six layers formed combining three bilayers of $\mathrm{LiF}$ and $\mathrm{MoO}_{3}$, the maximum $J_{\text {sc }}$ predicted corresponds to $72 \%$ that of the opaque cell. This confirms the relevance of increasing the degrees of freedom to reach a high level of tailoring for the EQE and, eventually, improve the performance of the device. As shown in Fig. 2b, when we compare the calculated EQE of the PC-Tn-Ag and Tn-Ag cells we observe that contributions to the current from the near-infrared as well as near-ultraviolet photons are clearly enhanced for the PC-Tn-Ag device. For some near-infrared photons the EQE for the PC-Tn-Ag is enhanced to such a level as to match the EQE of the opaque cell. On the other hand, the contribution from visible photons to the EQE remains similar to the contribution that we obtain from the same type of photons for the Tn-Ag cell. This results from the remarkable capacity of the photonic crystal to trap near-infrared and near-ultraviolet light, as can be seen in the field intensity contour plot in Fig. 4b. An enhanced field amplitude or localization is clearly observed in the near-ultraviolet and 

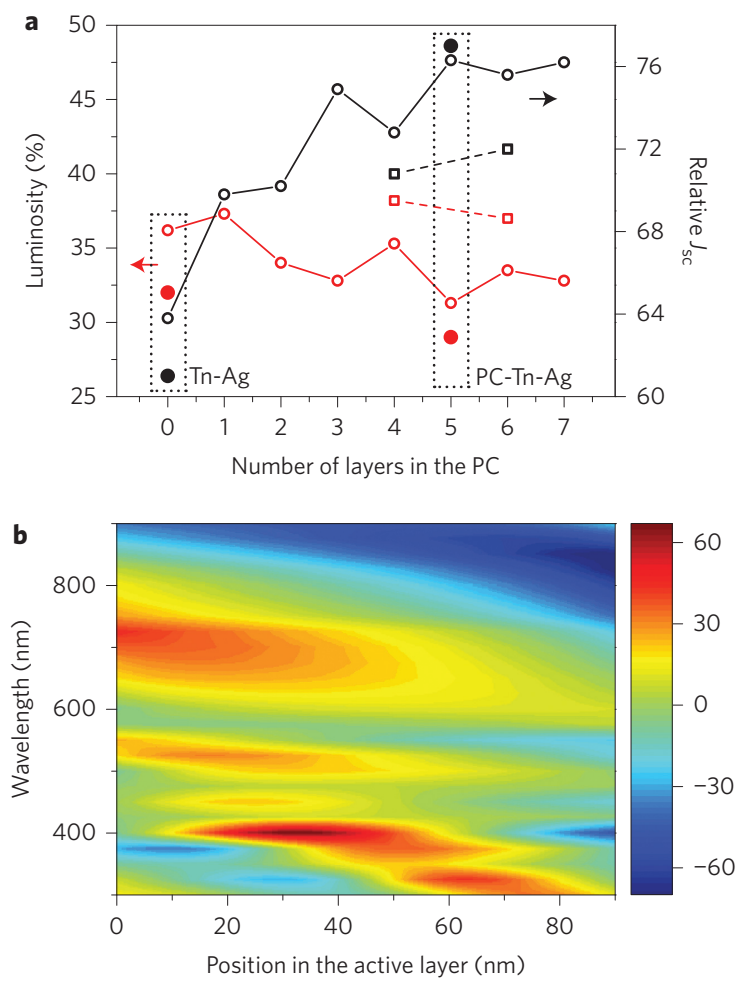

Figure 4 | Photonic crystal design. a, As a function of the number of layers, numerically determined short-circuit current (black open circles) and luminosity (red open circles) for devices incorporating the photonic crystal and short-circuit current (black open squares) and luminosity (red open squares) for devices incorporating the optimal periodic Bragg reflector. The experimentally measured short-circuit current (black filled circles) and luminosity (red filled circles) for the Tn-Ag and PC-Tn-Ag fabricated devices are also shown. Note that the zero in the $x$-axis corresponds to the case including a thin LiF protective layer. $\mathbf{b}$, Contour plot of the percentage change in the active layer field amplitude for the five-layer PC-Tn-Ag relative to the $\mathrm{Tn}-\mathrm{Ag}$ devices. The horizontal axis indicates the position in the active layer relative to the end of the PEDOT:PSS layer $(0 \mathrm{~nm})$ and the beginning of the BCP layer $(90 \mathrm{~nm})$.

near-infrared regions at several positions in the active layer, while in the visible region the field intensity change is minimal at almost all positions within this layer. The calculated transmissions (Fig. 3a) for the Tn-Ag and PC-Tn-Ag cells are in the 30-45\% range for most visible wavelengths. However, for near-ultraviolet and near-infrared wavelengths, the predicted transmission is below $10 \%$ for the PC-Tn-Ag, whereas it stays above $30 \%$ for the Tn-Ag cell.

To fabricate the photonic crystal we followed a procedure (see Methods) that imposed no degradation on the parameters of the cell (such as fill factor (FF) and $V_{\mathrm{oc}}$, which are weakly dependent on light-harvesting capacity). Indeed, as seen in Table 1 , the $V_{\mathrm{oc}}$ decreased from $724 \mathrm{mV}$ to $716 \mathrm{mV}$, and the FF from $71 \%$ to $68 \%$. The slight decrease in both parameters may be attributed to the silver electrode, which is only $10 \mathrm{~nm}$ thick. The experimentally measured EQE for the PC-Tn-Ag cell is compared to the Tn-Ag and Tk-Ag reference cells in Fig. 2a. In very good agreement with the theoretical prediction, the near-ultraviolet and near-infrared photon trapping is very effective. Indeed, the $J_{\mathrm{sc}}$ resulting from photons in the 300-400 $\mathrm{nm}$ wavelength range corresponds to $98 \%$ of the original opaque cell, while for the near-infrared photons in the $650-900 \mathrm{~nm}$ wavelength range the $J_{\mathrm{sc}}$ corresponds to $91 \%$ that of the original opaque cell. Experimentally, the overall increase in $J_{\text {sc }}$ relative to the Tn-Ag cell is close to $26 \%$, while transparency in the visible is maintained close to $30 \%$. The maximum efficiency
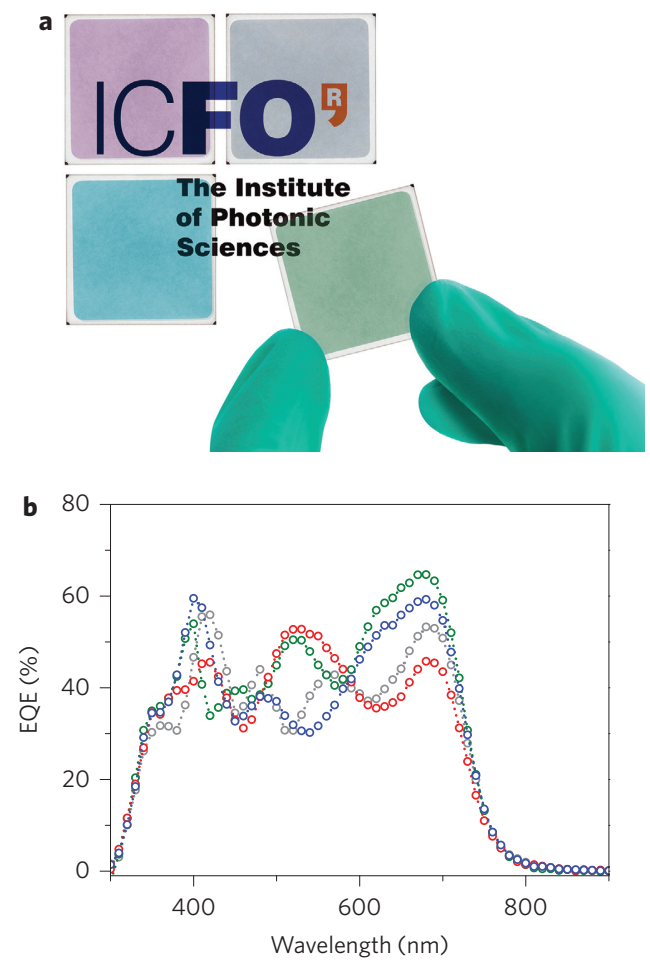

Figure 5 | Device colour control. a, Four PC-Tn-Ag devices fabricated with different relative layer thickness to tune the colour of the device. The ICFO logo is located under the devices, and illumination is provided from behind the logo. The size of each device in the image was $2.5 \mathrm{~cm} \times 2.5 \mathrm{~cm}$. The purpose of this is to show the colour tuning effect provided by the photonic crystal. Although the entire sequence of layers was deposited, no special design was made for the extraction of charges. The sequence and thicknesses of all the layers from the ITO to the protective LiF layer is the same in all four cases. All devices were covered with a five-layer photonic crystal, where the only difference between one photonic crystal and another is the relative thicknesses of the photonic-crystal layers. b. Experimentally measured EQEs for four devices equivalent to those in $\mathbf{a}$.

for a semi-transparent cell could be further increased using $120 \mathrm{~nm}$ ITO substrates as electrodes. For such a device, as summarized in Table 1, we measured a PCE of $5.6 \%$ for a cell device with a luminosity very close to $30 \%$. The $J_{\text {sc }}$ of the transparent cell was $77 \%$ that of the opaque device.

When looking through a thin layer of PTB7:PC ${ }_{71} \mathrm{BM}$ blend, one does not perceive any significant alteration of the colour of any image behind. Combining such fairly flat transmission (cf. Fig. 1a) with the photonic crystal, we demonstrated that we can broadly tune the colour of the device with a very limited PCE alteration. As explained in detail in Supplementary Section S2, from the predicted transmission curve of a given device one can predict its colour. We fabricated four different devices: green, blue, red and colourless (Fig. 5a). As reported in Supplementary Table S1, optimal harvesting of the near-infrared and near-ultraviolet photons is obtained with the blue and green devices (see EQE curves in Fig. 5b). For the red device, transmission for long wavelengths increases, resulting in less effective trapping of near-infrared photons (cf. Fig. 5b). The EQE of the colourless device shows the weakest wavelength dependence in the visible and nearinfrared intervals.

To summarize, in organic transparent cells, light harvesting diminishes because the reflectivity of the top layer is reduced and the device loses its capacity for photon trapping. We have opened a route to effective light-harvesting recovery when thinning down 
the top electrode. For the types of device considered here, a semitransparent top electrode implies that light-harvesting capacity is only $61 \%$ that of the corresponding opaque device. We demonstrate that the photon management provided by a non-periodic onedimensional structure of five layers is sufficient to return the light-harvesting capacity of the device to $77 \%$ that of the opaque cell. When such photon trapping was applied to high-performance bulk heterojunctions of OPV materials, we fabricated close to $30 \%$ transparent cells with efficiencies above $5.6 \%$. In a recent study of the theoretical efficiency limits of transparent cells it was determined that cells with $30 \%$ luminosity have the potential to exhibit a PCE close to $88 \%$ that of the Shockley-Queisser limit ${ }^{46}$. Combining the photonic control proposed here with the use of redshifted absorption, low-bandgap polymers, we expect that the performance of semi-transparent cells may come very close to such theoretical predictions. Finally, the results reported, which are essentially based on a photonic effect, have interest well beyond the photonics community. They open the door to the design and fabrication of new types of photovoltaic modules with great potential to be incorporated into buildings (Supplementary Section S3) as windows, thereby leading to very good integration of electrical power generation sources in highly populated urban areas.

\section{Methods}

Fabrication of photovoltaic cells. To fabricate the devices we used two different kinds of ITO-patterned substrates, one with 120-nm-thick ITO and another with 330 -nm-thick ITO. The thinner ITO provides a better performance in terms of photovoltaic parameters, but with significantly larger inhomogeneity in the relative performance of cells within the same substrate. Other details on the preparation of the Tk-Ag device can be found elsewhere ${ }^{48}$

In Supplementary Table S3, the specific architectures of the fabricated devices are provided. Note that all devices use the same thickness for the PTB7:PC ${ }_{71} B M$ active layer. This indicates that the optical extinction coefficient (or absorption of the active material) plays a secondary role in the final form of the EQEs (cf. Fig. 2), which exhibit significantly different features that can be directly linked to the field distribution imposed by the specific architecture in each case.

Design and fabrication of the photonic nanolayer structure. Given the simplicity of the layer architecture of an OPV cell, the numerical inverse integration method we chose here computed the outcome of all possible solutions for a given range of parameters (which essentially include the number of layers and thickness for some or all layers) and then numerically selected the solution that best matched the objective or target solution (set in terms of one or several variables, including shortcircuit current, average transparency at a given wavelength range, colour or luminosity of the devices). When the optical extinction coefficient and refractive index for all the layers, including the substrate, are known, it is straightforward, using a transfer matrix method ${ }^{49}$, to determine numerically the EQE (Fig. $2 \mathrm{~b}$ ) and transmission (Fig. 3b). From this point, all other required variables, such as $J_{\mathrm{sc}}$ and luminosity, can be calculated.

The fabricated photonic-crystal structure comprises a non-periodic alternation of low- and high-index-refraction layers with thicknesses of $\sim 100 \mathrm{~nm}$, as shown schematically in Fig. $1 \mathrm{~b}$ and Supplementary Table S13. The fabrication of such a photonic-crystal structure requires the use of methods and materials that do not introduce damage or degradation to the electrical properties of the cell underneath. Accordingly, the photonic multilayer structure was fabricated by thermal evaporation of low-refractive-index $(\mathrm{LiF})$ and high-refractive-index $\left(\mathrm{MoO}_{3}\right)$ materials. The photonic-crystal and semi-transparent back electrode of $10 \mathrm{~nm}$ of silver were deposited using a thermal evaporation system (Mini SPECTRO, Kurt J. Lesker Company). The deposition rate for silver was $5.5 \AA^{-1}$ and was carried out in a home-made cooled holder to decrease silver surface diffusion and therefore prevent three-dimensional island formation by alteration of the standard nucleation process $^{33}$. The silver electrode was deposited using masks made with laser beam cutting technology, which yielded well-defined areas. The deposition rate for the photonic-crystal materials was $1 \mathrm{~A}^{-1}$. Pellets/stones were used as material for evaporation. The residual vacuum pressure was kept below $1 \times 10^{-6}$ torr to prevent contamination. The photonic crystal was deposited close to normal incidence to prevent any optical problem related to a refractive index decrease due to an increment in the porosity of the film. The thicknesses of all layers were monitored using a crystal oscillator during deposition, and were later verified from transmission curves adjusted using an electromagnetic field transmission model. To improve electrical contact with the top thin silver layer, a further $100 \mathrm{~nm}$ layer of silver was deposited on top of the part of the silver contact not covered by the photonic crystal.

Note that the FF and $V_{\text {oc }}$ (Table 1) are significantly better for the PC-Tn-Ag cell than for the Tn-Ag cell. This, however, should be attributed to the rapid degradation of the Tn-Ag cell, which is less protected from the effects of oxygen or moisture because it lacks a photonic crystal on top. The devices were not encapsulated and all measurements were performed in air.

Measuring the performance of photovoltaic devices. The PCE of the fabricated devices was determined from current density-voltage curve measurements obtained under 1 sun, AM 1.5G spectrum illumination from a solar simulator (Abet Technologies, model Sun 3000). The solar simulator illumination intensity was monitored using a monocrystal silicon reference cell (Rera Systems) calibrated against a National Renewable Energy Laboratory calibrated reference cell. In all measurements of the semi-transparent cells, illumination was from the ITO side. EQE values were measured using a QEX10 Quantum Efficiency Measurement System (PV Measurements). For EQE measurements, the devices were illuminated using monochromatic light from a xenon lamp passing through a monochromator. A calibrated mono-silicon diode with known spectral response was used as a reference. The light transmission spectra for the fabricated devices were recorded using an ultraviolet-vis-near-infrared spectrophotometer (Lambda950, PerkinElmer).

Received 21 December 2012; accepted 10 September 2013; published online 20 October 2013

\section{References}

1. Bailey-Salzman, R. F., Rand, B. P. \& Forrest, S. R. Semitransparent organic photovoltaic cells. Appl. Phys. Lett. 88, 233502 (2006).

2. Ng, G.-M. et al. Optical enhancement in semitransparent polymer photovoltaic cells. Appl. Phys. Lett. 90, 103505 (2007).

3. Chen, F.-C., Wu, J.-L., Hsieh, K.-H., Chen, W.-C. \& Lee, S.-W. Polymer photovoltaic devices with highly transparent cathodes. Org. Electron. 9, 1132-1135 (2008)

4. Huang, J., Li, G. \& Yang, Y. A semi-transparent plastic solar cell fabricated by a lamination process. Adv. Mater. 20, 415-419 (2008).

5. Koeppe, R. et al. Organic solar cells with semitransparent metal back contacts for power window applications. ChemSusChem 2, 309-313 (2009).

6. Meiss, J. et al. Efficient semitransparent small-molecule organic solar cells. Appl Phys. Lett. 95, 213306 (2009).

7. Schmidt, H. et al. Efficient semitransparent inverted organic solar cells with indium tin oxide top electrode. Appl. Phys. Lett. 94, 243302 (2009).

8. Tanaka, S. et al. Semitransparent organic photovoltaic cell with carbon nanotube-sheet anodes and Ga-doped $\mathrm{ZnO}$ cathodes. Synth. Metals 159, 2326-2328 (2009)

9. Bedeloglua, A., Demirb, A., Bozkurta, Y. \& Sariciftci, N. S. Photovoltaic properties of polymer based organic solar cells adapted for non-transparent substrates. Renew. Energy 35, 2301-2306 (2010).

10. Ameri, T. et al. Fabrication, optical modeling, and color characterization of semitransparent bulk-heterojunction organic solar cells in an inverted structure. Adv. Funct. Mater. 20, 1592-1598 (2010)

11. Dong, Q. et al. All-spin-coating vacuum-free processed semi-transparent inverted polymer solar cells with PEDOT:PSS anode and PAH-D interfacial layer. Org. Electron. 11, 1327-1331 (2010).

12. Lee, J.-Y., Connor, S. T., Cui, Y. \& Peumans, P. Semitransparent organic photovoltaic cells with laminated top electrode. Nano Lett. 10, 1276-1279 (2010).

13. Nickel, F. et al. Cathodes comprising highly conductive poly(3,4-ethylenedioxythiophene):poly(styrenesulfonate) for semi-transparent polymer solar cells. Org. Electron. 11, 535-538 (2010).

14. Pandey, A. K. \& Samuel, I. D. W. Photophysics of solution-processed transparent solar cells under top and bottom illumination. IEEE J. Sel. Topics Quantum Electron. 16, 1560-1564 (2010).

15. Zhou, Y. et al. Indium tin oxide-free and metal-free semitransparent organic solar cells. Appl. Phys. Lett. 97, 153304 (2010).

16. Wang, X., Ng, G.-M., Ho, J.-W., Tam, H.-L. \& Zhu, F. Efficient semitransparent bulk-heterojunction organic photovoltaic cells with high-performance low processing temperature indium-tin oxide top electrode. IEEE J. Sel. Topics Quantum Electron. 16, 1685-1689 (2010).

17. Colsmann, A. et al. Efficient semi-transparent organic solar cells with good transparency color perception and rendering properties. Adv. Energy Mater. 1, 599-603 (2011).

18. Lee, Y.-Y. et al. Top laminated graphene electrode in a semitransparent polymer solar cell by simultaneous thermal annealing/releasing method. ACS Nano 5, 6564-6570 (2011).

19. Lewis, J. E., Lafalce, E., Toglia, P. \& Jiang, X. Over 30\% transparency large area inverted organic solar array by spray. Solar Energy Mater. Solar Cells 95, 2816-2822 (2011)

20. Meiss, J., Holzmueller, F., Gresser, R., Leo, K. \& Riede, M. Near-infrared absorbing semitransparent organic solar cells. Appl. Phys. Lett. 99, 193307 (2011).

21. Peh, R. J., Lu, Y., Zhao, F., Lee, C.-L. K. \& Kwan, W. L. Vacuum-free processed transparent inverted organic solar cells with spray-coated PEDOT: PSS anode. Solar Energy Mater. Solar Cells 95, 3579-3584 (2011). 
22. Bauer, A., Wahl, T., Hanisch, J. \& Ahlswede, E. ZnO: Al cathode for highly efficient, semitransparent $4 \%$ organic solar cells utilizing $\mathrm{TiO}_{x}$ and aluminum interlayers. Appl. Phys. Lett. 100, 073307 (2012).

23. Colsmann, A. et al. Inverted semi-transparent organic solar cells with spray coated, surfactant free polymer top-electrodes. Solar Energy Mater. Solar Cells 98, 118-123 (2012).

24. Kang, J.-W. et al. All-spray-coated semitransparent inverted organic solar cells from electron selective to anode layers. Org. Electron. 13, 2940-2944 (2012).

25. Kim, Y. H. et al. Semi-transparent small molecule organic solar cells with laminated free-standing carbon nanotube top electrodes. Solar Energy Mater. Solar Cells 96, 244-250 (2012).

26. Tang, Z. et al. Semi-transparent tandem organic solar cells with $90 \%$ internal quantum efficiency. Adv. Energy Mater. 2, 1467-1476 (2012).

27. Kim, H. P., Lee, H. J., Yusoff, A. R. B. M. \& Jang, J. Semi-transparent organic inverted photovoltaic cells with solution processed top electrode. Solar Energy Mater. Solar Cells 108, 38-43 (2013).

28. Tao, C. et al. Semitransparent inverted polymer solar cells with $\mathrm{MoO}_{3} / \mathrm{Ag} / \mathrm{MoO}_{3}$ as transparent electrode. Appl. Phys. Lett. 95, 053303 (2009).

29. Shen, L. et al. Semitransparent polymer solar cells using $\mathrm{V}_{2} \mathrm{O}_{5} / \mathrm{Ag} / \mathrm{V}_{2} \mathrm{O}_{5}$ as transparent anodes. Org. Electron. 12, 1223-1226 (2011).

30. Tao, C., Xie, G., Meng, F., Ruan, S. \& Chen, W. Tailoring spatial distribution of the optical field intensity in semitransparent inverted organic solar cells. J. Phys. Chem. C 115, 12611-12615 (2011).

31. Winkler, T. et al. Efficient large area semitransparent organic solar cells based on highly transparent and conductive ZTO/Ag/ZTO multilayer top electrodes. Org. Electron. 12, 1612-1618 (2011).

32. Jin, H. et al. Efficient large area ITO-and-PEDOT-free organic solar cell submodules. Adv. Mater. 24, 2572-2577 (2012).

33. Sergeant, N. P. et al. Design of transparent anodes for resonant cavity enhanced light harvesting in organic solar cells. Adv. Mater. 24, 728-732 (2012).

34. Liu, Z. et al. The application of highly doped single-layer graphene as the top electrodes of semitransparent organic solar cells. ACS Nano 6, 810-818 (2012).

35. Krantz, J. et al. Spray-coated silver nanowires as top electrode layer in semitransparent P3HT:PCBM-based organic solar cell devices. Adv. Funct. Mater. 23, 1711-1717 (2013).

36. Guo, F. et al. ITO-free and fully solution-processed semitransparent organic solar cells with high fill factors. Adv. Energy Mater. 3, 1062-1067 (2013).

37. Lunt, R. R. \& Bulovic, V. Transparent, near-infrared organic photovoltaic solar cells for window and energy-scavenging applications. Appl. Phys. Lett. 98, 113305 (2011).

38. Yu, W. et al. Simultaneous improvement in efficiency and transmittance of low bandgap semitransparent polymer solar cells with one-dimensional photonic crystals. Solar Energy Mater. Solar Cells 117, 198-202 (2013).

39. O’Brien, P. G. et al. Selectively transparent and conducting photonic crystal rear-contacts for thin-film silicon-based building integrated photovoltaics. Opt. Express 19, 17040 (2011).
40. Galagan, Y., Debije, M. G. \& Blom, P. W. M. Semitransparent organic solar cells with organic wavelength dependent reflectors. Appl. Phys. Lett. 98, 043302 (2011)

41. Chen, C.-C. et al. Visibly transparent polymer solar cells produced by solution processing. ACS Nano 6, 7185-7190 (2012)

42. Dou, L. et al. A selenium-substituted low-bandgap polymer with versatile photovoltaic applications. Adv. Mater. 25, 825-831 (2013).

43. Meiss, J. et al. Highly efficient semitransparent tandem organic solar cells with complementary absorber materials. Appl. Phys. Lett. 99, 043301 (2011).

44. Chen, K.-S. et al. Semi-transparent polymer solar cells with 6\% PCE, 25\% average visible transmittance and a color rendering index close to 100 for power generating window applications. Energy Environ. Sci. 5, 9551-9557 (2012).

45. Chueh, C.-C. et al. Toward high-performance semi-transparent polymer solar cells: optimization of ultra-thin light absorbing layer and transparent cathode architecture. Adv. Energy Mater. 3, 417-423 (2013).

46. Lunt, R. R. Theoretical limits for visibly transparent photovoltaics. Appl. Phys. Lett. 101, 043902 (2012).

47. He, Z. et al. Enhanced power-conversion efficiency in polymer solar cells using an inverted device structure. Nature Photon. 6, 591-595 (2012).

48. Martínez-Otero, A., Elias, X., Betancur, R. \& Martorell, J. High performance polymer solar cells using an optically enhanced architecture. Adv. Opt. Mater. 1, 37-42 (2013).

49. Betancur, R. et al. Optical interference for the matching of the external and internal quantum efficiencies in organic photovoltaic cells. Solar Energy Mater. Solar Cells 104, 87-91 (2012).

\section{Acknowledgements}

The authors acknowledge support from the Ministerio de Economía y Competitividad (grants MAT2011-28665, IPT-120000-2010-29, IPT-2012-0986-120000 and CSD2007-00046)

\section{Author contributions}

R.B. and P.R.-G. contributed equally in the optical simulation, design and fabrication of the transparent organic cells, including the non-periodic photonic crystal. R.B., P.R.-G., A.M.-O. and X.E. contributed to the optimization and fabrication of the organic devices. R.B., P.R.-G., A.M.-O. and M.M. contributed to the fabrication of the transparent electrode. The project was planned and supervised by J.M. The manuscript was written by J.M. with the assistance of all other authors.

\section{Additional information}

Supplementary information is available in the online version of the paper. Reprints and permissions information is available online at www.nature.com/reprints. Correspondence and requests for materials should be addressed to J.M.

\section{Competing financial interests}

The authors declare no competing financial interests. 